\title{
Dyslipidaemia was correlated to the posterior circulation infarction in non-diabetic populations
}

\author{
Yun Luo ${ }^{1 * \dagger}$, Zheng $\mathrm{Li}^{2 \dagger}$, Jiahui Zhang ${ }^{1}$, Jingwei $\mathrm{Li}^{1}$ and Zhengjuan Lu'
}

\begin{abstract}
Background: Diabetes mellitus (DM) was prone to happening in posterior circulation infarction (POCI) and DM also has the impact on the lipids, our study was to investigate the correlation between lipid compositions and POCI.

Methods: Data was collected from the patients with acute ischemic stroke (AIS) hospitalization in Affiliated Drum Tower Hospital of Nanjing University Medical School from October 2008 to May 2012. Lipids and other risk factors in the different populations were investigated in relation to occurrence of POCI based on the infarction location.

Results: Six hundred ten patients with AIS were included in this study, which had 428 with anterior circulation infarction (ACl) and 182 with POCI. Elevated Triglyceride (TG) and decreased High density lipoprotein cholesterol $(\mathrm{HDL}-\mathrm{C})$ were seen in the POCl of total populations and AIS without DM compared to the $\mathrm{ACl}$, but not in the populations of AIS with DM, so did the elevated TG/HDL-C ratios. Also, the percent of low HDL-C level and high TG level were higher in $\mathrm{POCl}$ group than that in $\mathrm{ACl}$ group. Furthermore, single factors logistic regression demonstrated that TG, HDL-C and TG/HDL-C ratio were correlated to the POCl whatever in the total populations or AIS without DM, but this kind of trend just maintained in the populations of AIS without DM after adjusting by relative interference factors.
\end{abstract}

Conclusion: Dyslipidaemia was prone to happening in $\mathrm{POCl}$ compared to ACl in the non-diabetic populations, which was correlated to the pathogenesis of POCl.

Keywords: Triglyceride, High density lipoprotein cholesterol, Acute ischemic stroke, Posterior circulation infarction, Diabetes mellitus

\section{Background}

Acute ischemic stroke (AIS) was divided into anterior circulation infarction (ACI) and posterior circulation infarction (POCI) according to the infarction location, a difference was made between ACI and POCI in terms of clinical manifestations and prognosis. Despite POCI just accounting for $20 \%$ of AIS, and it would have a relatively good outcome, but it had high rate of recurrence. About the risk factors, was there has any difference between $\mathrm{ACI}$ and POCI, the conclusions was inconsistent.

\footnotetext{
* Correspondence: njluoyun@sina.com

${ }^{\dagger}$ Yun Luo and Zheng Li contributed equally to this work.

'Department of Neurology, Affiliated Drum Tower Hospital of Nanjing

University Medical School, No. 321 Zhongshan Road, Gulou District, Nanjing

210008, Jiangsu Province, China

Full list of author information is available at the end of the article
}

As the important risk factors of AIS, diabetes mellitus (DM) had obvious impact on both of subtypes, but DM was more prone to linked with POCI [1], which was identified in many studies include ours (Article in Chinese) [2-4]. A study from China demonstrated, not only diabetes mellitus, but also male gender was associated with greater likelihood of POCI than ACI [5].

Diabetes mellitus had obvious impact on the lipids, which mainly reflects in the rise of Triglyceride (TG) and fall of High density lipoprotein cholesterol (HDL-C) [6, 7]. Both the blood glucose and lipids would play the important role on the occurrence of arteriosclerosis, while lipids would play the more direct role $[8,9]$. Did this mean that the induction of arteriosclerosis and ischemic stroke by DM was through lipids, and this was proved in our previous study, that was, low level of HDL-C was correlated to 
the occurrence of AIS induced by DM [10], which demonstrated that dyslipidaemia was prone to happening in the AIS with DM.

There was more percentage of DM in the POCI, also, DM would have the impact on the expression of lipids. Based on the background above, we wonder if there would have more dyslipidaemia in the POCI compared to the ACI, which maybe act as the important pathogenic factors of POCI.

\section{Methods}

\section{Study subjects}

Data for this retrospective study was collected from the hospitalization patients of department of neurology in Affiliated Drum Tower Hospital of Nanjing University Medical School from October 2008 to May 2012, AIS was defined as symptom onset within7 days. The study was approved by our institutional committee. Patients who were found with pre-stroke impairment or insulin-dependent diabetes mellitus were excluded. At admission, plain CT scan of the head was done to rule out haemorrhage and MRI was done to identify the new infarction and the location of the lesion, otherwise such patients would also be excluded. The stroke subtype (ACI and POCI) was defined based on the classification of Bogousslavsky's [11].

\section{Definition of vascular risk factors}

Hypertension and DM were defined as participants with history of relative disease or new diagnosis according to the China hypertension and DM standard (just non-insulin-dependent diabetes were included), while atrial fibrillation (AF) was defined as participants with history of AF or new diagnosis by electrocardiogram.

\section{Blood collection and analysis}

Venous blood was collected following overnight fasting for at least $12 \mathrm{~h}$, and analyzed by a solid-phase chemiluminescent immunometric assay on Immulite 2000 with the manufacturer's reagents as directed to detect total bilirubin (Tbil), direct bilirubin (Dbil), blood glucose (BG), uric acid (UA), TG, total cholesterol (TC), HDL-C, Low density lipoprotein cholesterol (LDL-C).

\section{Statistical analyses}

Statistical analyses were performed with SPSS 17.0 software. The results are expressed as constituent ratio for categorical variables ( $x 2$ test) and as mean \pm SEM for the continuous variables (t-test) depending on their normal distribution. The level-risk relationship was expressed as an OR, with a corresponding 95\% CI, through logistic regression. Level of significance for statistical purposes was stated at $p<0.05$.

\section{Results}

Baseline characteristics

Six hundred ten patients with AIS were included in the trial, among them, 385 were male and 225 were female, whose age range from 15 to 92 . There were 202 patients who had DM coexisted with AIS. Infarction happened to the ACI was 428, and POCI was 182.

\section{Risk factors of $\mathrm{POCl}$ compared to the $\mathrm{ACl}$}

There was $29 \%$ of DM in the ACI, while $42.9 \%$ in the POCI, the difference was significant $(P=0.001)$, the same trend was existed in hypertension and BG. While $\mathrm{AF}$ and Dbil were more prevalent in ACI. Also, the difference of TG and HDL-C between ACI and POCI was significant, as shown in Table 1.

\section{Comparison of lipids level between $\mathrm{ACl}$ and $\mathrm{POCl}$ in the populations of AIS with and without DM}

DM would have the impact on express of lipids [12], which had been proved in a series of studies include ours [13]. Due to more DM coexisted with POCI, we wonder if this kind of distribution difference resulted in difference of the lipids between ACI and POCI. But to our surprised, elevated TG and decreased HDL-C were just seen in the populations of AIS without DM, while not in the AIS with DM (Table 2).

\section{Comparison of lipids distribution between the $\mathrm{ACl}$ and $\mathrm{POCl}$}

HDL cholesterol concentrations were grouped into 3 levels: < 1.03, 1.03-1.53 and > $1.53 \mathrm{mmol} / \mathrm{l}$. We analyzed the difference of distribution of HDL-C between the POCI and ACI whenever in the total or AIS without DM populations, and found that, the percent of low

Table 1 Comparison of risk factors between $\mathrm{ACl}$ and $\mathrm{POCl}$

\begin{tabular}{llll}
\hline Variable & $\mathrm{ACl}$ & $\mathrm{POCl}$ & $P$ \\
\hline Male (\%) & 61.4 & 67.0 & 0.200 \\
Age & $67.131 \pm 0.633$ & $65.758 \pm 0.935$ & 0.232 \\
DM (\%) & 29.0 & 42.9 & 0.001 \\
Hypertension (\%) & 67.8 & 79.1 & 0.005 \\
AF (\%) & 15.7 & 7.7 & 0.009 \\
Dbil & $4.867 \pm 0.130$ & $4.329 \pm 0.156$ & 0.017 \\
Tbil & $18.638 \pm 0.462$ & $17.548 \pm 0.674$ & 0.192 \\
BG & $6.758 \pm 0.131$ & $7.297 \pm 0.227$ & 0.041 \\
UA & $326.348 \pm 4.751$ & $335.524 \pm 8.334$ & 0.313 \\
CRP & $5.448 \pm 0.873$ & $3.272 \pm 0.831$ & 0.072 \\
TG & $1.450 \pm 0.922$ & $1.641 \pm 1.116$ & 0.028 \\
TC & $4.795 \pm 0.981$ & $4.809 \pm 1.142$ & 0.877 \\
HDL-C & $1.170 \pm 0.367$ & $1.082 \pm 0.346$ & 0.006 \\
LDL-C & $2.549 \pm 0.733$ & $2.595 \pm 0.779$ & 0.485 \\
\hline
\end{tabular}


Table 2 Comparison of lipid composition between $\mathrm{ACl}$ and $\mathrm{POCl}$

\begin{tabular}{llll}
\hline Variables & $\mathrm{ACl}(n=124)$ & $\mathrm{POCl}(n=78)$ & $P$ value \\
\hline With DM & & & \\
$\mathrm{TG}(\mathrm{mmol} / \mathrm{l})$ & $1.555 \pm 1.021$ & $1.623 \pm 0.799$ & 0.617 \\
$\mathrm{TC}(\mathrm{mmol} / \mathrm{l})$ & $4.722 \pm 1.118$ & $4.858 \pm 1.169$ & 0.409 \\
$\mathrm{HDL}-\mathrm{C}(\mathrm{mmol} / \mathrm{l})$ & $1.076 \pm 0.327$ & $1.053 \pm 0.321$ & 0.629 \\
$\mathrm{LDL}-\mathrm{C}(\mathrm{mmol} / \mathrm{l})$ & $2.530 \pm 0.832$ & $2.687 \pm 0.810$ & 0.190 \\
Without DM & & & \\
$\mathrm{TG}(\mathrm{mmol} / \mathrm{l})$ & $1.407 \pm 0.877$ & $1.655 \pm 1.308$ & 0.030 \\
$\mathrm{TC}(\mathrm{mmol} / \mathrm{l})$ & $4.825 \pm 0.919$ & $4.773 \pm 1.126$ & 0.639 \\
$\mathrm{HDL}-\mathrm{C}(\mathrm{mmol} / \mathrm{l})$ & $1.209 \pm 0.375$ & $1.103 \pm 0.363$ & 0.013 \\
$\mathrm{LDL}-\mathrm{C}(\mathrm{mmol} / \mathrm{l})$ & $2.557 \pm 0.689$ & $2.526 \pm 0.752$ & 0.707 \\
\hline
\end{tabular}

HDL-C level was higher in POCI group than that in ACI group. Also, TG concentrations were grouped into 2 levels: $<1.7$ and $>1.7 \mathrm{mmol} / \mathrm{l}$, we found, the percent of high TG level was higher in POCI group than that in ACI group both in the total or AIS without DM populations (Fig. 1).

\section{Difference of the ratio of TG/HDL-C between $\mathrm{ACl}$ and POCI}

Dyslipidaemia in the POCI was mainly resulted from the change of TG and HDL-C, while the ratio of TG/HDL-C could be used as the marker in prognosis of vascular events [14], so we compared the ratio of TG/HDL-C between ACI and POCI. We found that TG/HDL-C ratios was higher in the POCI compared to the ACI both in the total and non-diabetic populations, which demonstrated that dyslipidaemia played the more important role on POCI (Fig. 2).

\section{Dyslipidaemia was not correlated to POCI in the total AIS populations}

All the patients of AIS, were divided into two groups named ACI and POCI based on the location of infarction. To study the risk factors of POCI, we first performed the single-factor logistic regression, and found that TG, HDL-C and TG/HDL-C ratio were correlated to the POCI, but after multivariable logistic regression analysis, the correlation was disappeared (Table 3).

\section{Dyslipidaemia was correlated to the POCI in the AIS without DM populations}

Populations of AIS without DM were also divided into $\mathrm{ACI}$ and POCI, logistic regression demonstrated that TG, HDL-C and TG/HDL-C ratio were correlated to the POCI, whatever in the single-factor or multivariable logistic regression (Table 4).

\section{Discussion}

Our investigation was a retrospective study of patients with AIS in which the correlation between POCI and lipids was evaluated. We first confirmed that there was more percentage of DM in the POCI compared to the $\mathrm{ACI}$, while DM would have the impact on the expression
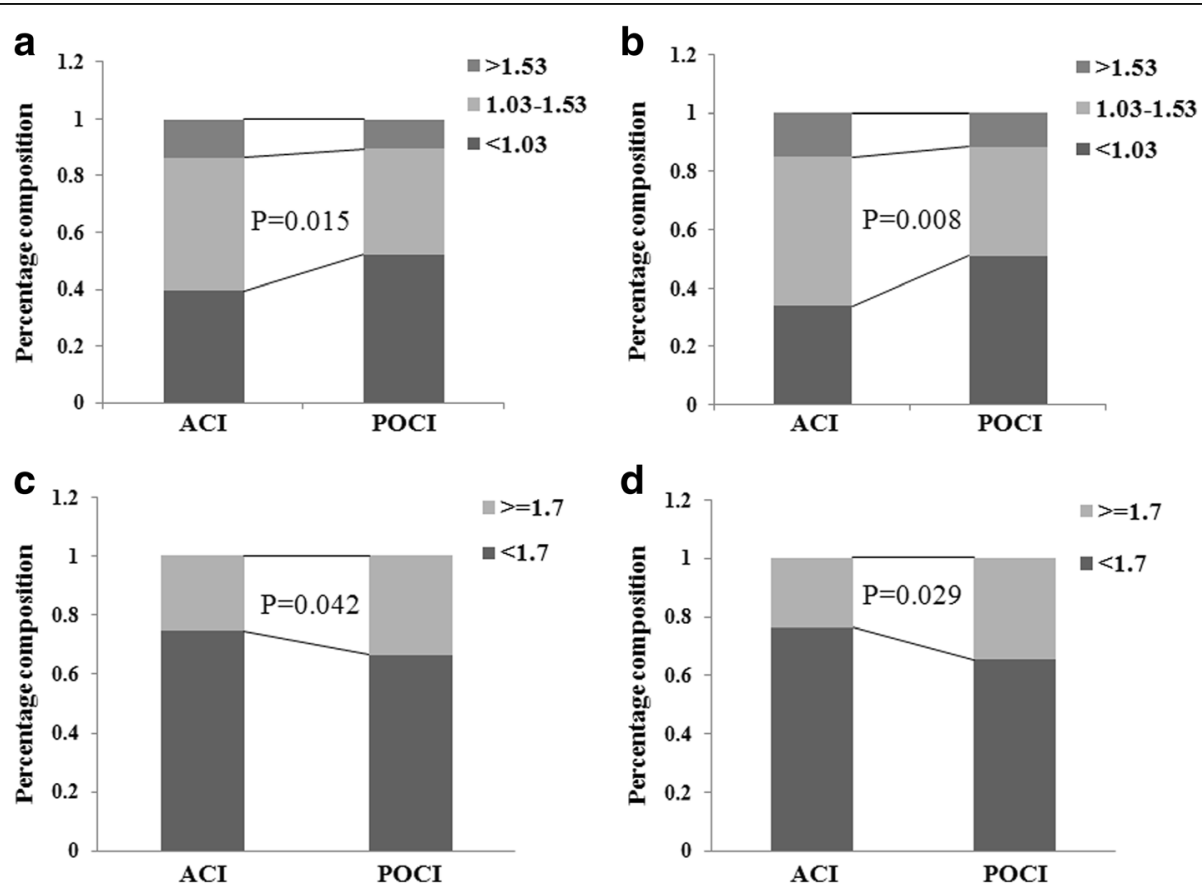

Fig. 1 Distribution of HDL-C and TG levels in the total and non-DM populations. $\mathbf{a} H D L-C$ in the total populations, $\mathbf{b} H D L-C$ in the non-DM populations, c TG in the total populations, $\mathbf{d}$ TG in the non-DM populations) 


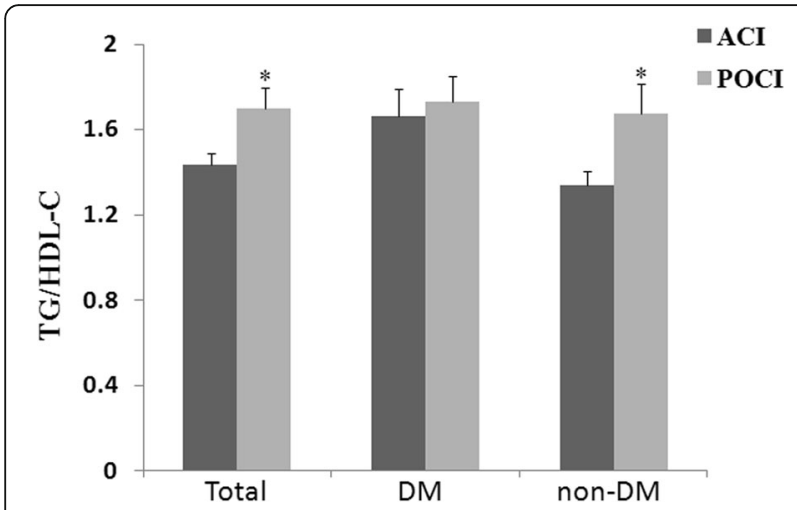

Fig. 2 Comparison of TG/HDL-C ratios between $A C l$ and $P O C l$ in different populations $\left({ }^{*} P<0.05\right.$ vs $\left.A C l\right)$

of lipids, which has been identified in our previous study [13]. We hypothesize that this kind of distribution difference would result in difference of lipids between ACI and POCI, but this hypothesis was uncorrected. In fact, the significant difference of TG, HDL, TG/HDL-C between ACI and POCI were just existed in the populations of non-diabetic AIS, which was correlated to the pathogenesis of POCI.

The association between DM and POCI has been proved in previous studies $[15,16]$. In a prospective study in Koreans, DM was an independent risk factor for intracranial atherosclerosis only in posterior circulation [2]. These researchers speculated that the effect of metabolic

Table 3 Relative risk of $\mathrm{POCl}$ versus risk factors in the total populations of AIS

\begin{tabular}{lllll}
\hline Variables & Beta estimate & OR & $95 \% \mathrm{Cl}$ & $P$ value \\
\hline Model 1: unadjusted & & & & \\
Male & 0.243 & 1.276 & $0.885-1.838$ & 0.191 \\
Age & -0.008 & 0.992 & $0.979-1.005$ & 0.231 \\
DM & 0.609 & 1.839 & $1.282-2.636$ & 0.001 \\
Hypertension & 0.590 & 1.803 & $1.196-2.720$ & 0.005 \\
AF & -0.801 & 0.449 & $0.245-0.822$ & 0.009 \\
Dbil & -0.104 & 0.902 & $0.828-0.981$ & 0.017 \\
Tbil & -0.013 & 0.987 & $0.968-1.007$ & 0.193 \\
UA & 0.001 & 1.001 & $0.999-1.003$ & 0.313 \\
TG & 0.186 & 1.204 & $1.015-1.428$ & 0.033 \\
TC & 0.013 & 1.013 & $0.856-1.199$ & 0.876 \\
HDL-C & -0.743 & 0.476 & $0.280-0.808$ & 0.006 \\
LDL-C & 0.083 & 1.086 & $0.862-1.369$ & 0.484 \\
TG/HDL-C & 0.169 & 1.184 & $1.033-1.357$ & 0.015
\end{tabular}

Model 2: adjusted for Hypertension, DM, AF, Dbil

\begin{tabular}{lllll} 
TG & 0.108 & 1.114 & $0.934-1.328$ & 0.229 \\
HDL & -0.522 & 0.594 & $0.343-1.028$ & 0.063 \\
TG/HDL-C & 0.097 & 1.102 & $0.956-1.271$ & 0.180 \\
\hline
\end{tabular}

Table 4 Relative risk of $\mathrm{POCl}$ versus risk factors in the populations of non-diabetic AIS

\begin{tabular}{lllll}
\hline Variable & \multicolumn{1}{l}{ Beta estimate } & OR & $95 \% \mathrm{Cl}$ & $P$ value \\
\hline Model 1: unadjusted & & & & \\
Male & 0.453 & 1.574 & $0.967-2.562$ & 0.068 \\
Age & -0.009 & 0.991 & $0.976-1.008$ & 0.296 \\
Hypertension & 0.626 & 1.870 & $1.127-3.102$ & 0.015 \\
AF & -0.436 & 0.647 & $0.322-1.300$ & 0.221 \\
Dbil & -0.105 & 0.901 & $0.808-1.004$ & 0.058 \\
Tbil & -0.017 & 0.983 & $0.958-1.008$ & 0.180 \\
BG & -0.022 & 0.978 & $0.846-1.131$ & 0.765 \\
UA & 0.001 & 1.001 & $0.999-1.003$ & 0.222 \\
TG & 0.222 & 1.248 & $1.011-1.541$ & 0.039 \\
TC & -0.055 & 0.946 & $0.752-1.191$ & 0.638 \\
HDL-C & -0.868 & 0.420 & $0.211-0.834$ & 0.013 \\
LDL-C & -0.061 & 0.941 & $0.685-1.292$ & 0.707 \\
TG/HDL-C & 0.220 & 1.247 & $1.044-1.488$ & 0.015 \\
Model 2: adjusted for Hypertension & & & \\
TG & 0.592 & 1.807 & $1.086-3.006$ & 0.023 \\
HDL & -0.809 & 0.445 & $0.221-0.895$ & 0.023 \\
TG/HDL-C & 0.203 & 1.225 & $1.024-1.466$ & 0.027 \\
\hline
\end{tabular}

disorders was more related to the POCI, which indicated the differing neurovascular origins of POCI and ACI [3]. In our research, we found that more DM coexisted with POCI compared to the ACI, which seemed to demonstrate that DM cause dyslipidaemia and finally increase the incidence of POCI.

Dyslipidaemia was prone to happening in the diabetic populations $[17,18]$, which played important role in the pathogenesis of AIS. Insulin resistance (IR) existed in many diabetic population and altered the lipids and lipoprotein metabolism $[6,19,20]$. Through a series of signaling pathways adjusting, the decrease in HDL available for participation in reverse cholesterol transport may finally caused the atherogenicity in AIS [21]. In the prevention of AIS linked to the DM, management of lipids was important as well as the glucose control [22]. Compared to the diabetic populations, what we should do in the non-diabetic populations? There did not involve the glucose control, but dyslipidaemia also act as an important risk factors, mainly in the POCI, which was identified in this study.

Low levels of HDL-C had been proved correlated with AIS [23-25], but the difference between POCI and ACI was unclear. In our study, elevated TG/HDL-C ratio was prone to happening in the POCI compared to the ACI, both in the total and non-diabetic populations, while not in the populations of DM. Why was this kind of manifest? We found, level of TG was higher and HDL-C was lower in the POCI of total and non-diabetic patients, resulting in higher TG/HDL-C ratios compared to the $\mathrm{ACI}$, while 
the level of TG and HDL-C had no difference between POCI and ACI in the diabetic patients.

Perhaps this kind of infarction location difference also shown difference of the expression of HDL-C and TG, but compared to the influence of DM, the former is negligible, and finally determined that there had no difference of HDL-C and TG between ACI and POCI in the diabetic patients. In order to confirm whether our hypothesis was right, we studied the risk factors of POCI in total and patients without DM.

We observed the elevated TG/HDL-C ratio in the POCI rooted from the rise of TG and fall of HDL-C, then, logistic regression demonstrated it was correlated to the POCI just in the non-diabetic populations, which indicated that elevated TG/ HDL-C ratio was an important risk factor in the pathogenesis of $\mathrm{POCI}$ in the non-diabetic populations. Elevated TG and decreased HDL-C were thought to be asatherogenic dyslipidaemia [26] and the key metabolic abnormalities in insulin resistance (IR) states [27], which was familiar in the patients of DM. This result we found perhaps predicate that more patients of POCI had been in the condition of pre-diabetes.

\section{Conclusion}

Our current study provided the evidence that high level of TG and low level of HDL-C, furthermore, elevated TG/ HDL-C ratio, were correlated to the occurrence of POCI in the non-diabetic populations. We would certainly give enough attentions to the dyslipidaemia in the diabetic patients [28], but to prevent the occurrence of ischemic stroke in the non-diabetic populations, more attentions should be paid to the change of the lipids including TG, HDL-C and TG/HDL-C ratio, which has the obvious correlation with POCI.

\section{Abbreviations}

ACl: Anterior circulation infarction; AF: Atrial fibrillation; AIS: Acute ischemic stroke; BG: Blood glucose; Dbil: Direct bilirubin; DM: Diabetes mellitus; HDLC: High density lipoprotein cholesterol; LDL-C: Low density lipoprotein cholesterol; POCI: Posterior circulation infarction; Tbil: Total bilirubin; TC: Total cholesterol; TG: Triglyceride; UA: Uric acid
}

\section{Acknowledgements \\ I would like to express my gratitude to all those who have helped me during the course of study and writing of this paper. I gratefully acknowledge the help of my colleagues who provided us enough research objects.}

\section{Funding}

This work was supported by National Natural Science Foundation of China (81671140) and Nanjing Medical Science and technique Development Foundation (QRX17002).

\section{Availability of data and materials}

The datasets used and/or analyzed during the current study are available from the corresponding author upon reasonable request.

\section{Authors' contributions}

$Y L, Z L$ and JWL designed the study and participated in acquisition of data; $\mathrm{YL}, J \mathrm{HZ}$, ZJL undertook the statistical analysis and wrote the manuscript. ZL revised the manuscript critically for important intellectual content and languages. All authors read and approved the final manuscript.

\section{Ethics approval and consent to participate}

This study was approved by the ethics committee of Affiliated Drum Tower Hospital of Nanjing University Medical School and conducted in accordance with the ethical principles of by the Declaration of Helsinki. Written informed consent was obtained from all patients.

\section{Consent for publication}

All co-authors and participants have given their consent for publication of this article in Lipids in Health and Disease.

\section{Competing interests}

The authors declare that they have no conflict of interests.

\section{Publisher's Note}

Springer Nature remains neutral with regard to jurisdictional claims in published maps and institutional affiliations.

\section{Author details}

${ }^{1}$ Department of Neurology, Affiliated Drum Tower Hospital of Nanjing University Medical School, No. 321 Zhongshan Road, Gulou District, Nanjing 210008, Jiangsu Province, China. ${ }^{2}$ Department of Rehabilitation Medicine, Affiliated Drum Tower Hospital of Nanjing University Medical School, No. 321 Zhongshan Road, Gulou District, Nanjing 210008, Jiangsu Province, China.

Received: 14 February 2018 Accepted: 12 June 2018

Published online: 26 June 2018

\section{References}

1. Rincon F, Sacco RL, Kranwinkel G, Xu Q, Paik MC, Boden-Albala B, Elkind MS. Incidence and risk factors of intracranial atherosclerotic stroke: thenorthern Manhattan stroke study. Cerebrovasc Dis. 2009;28:65-71.

2. Kim JS, Nah HW, Park SM, Kim SK, Cho KH, Lee J, Lee YS, Kim J, Ha SW, Kim EG, Kim DE, Kang DW, Kwon SU, Yu KH, Lee BC. Risk factors and stroke mechanisms in atherosclerotic stroke: intracranial compared with extracranial and anterior compared with posterior circulation disease. Stroke. 2012;43:3313-8

3. Subramanian G, Silva J, Silver FL, Fang J, Kapral MK, Oczkowski W, Gould L, O'Donnell MJ, Investigators of the Registry of the Canadian Stroke Network. Risk factors for posterior compared to anterior ischemic stroke: an observational study of the Registry of the Canadian Stroke Network. Neuroepidemiology. 2009;33:12-6.

4. Luo Y, Li J, Li Z, Zhang Y, Wu J, Wang Z, Wang C, Guan D, Xu Y. Imaging distribution characteristic of cerebral infarction with diabetes mellitus. J Chin Physician. 2016;18:998-1004.

5. Zeng Q, Tao W, Lei C, Dong W, Liu M. Etiology and risk factors of posterior circulation infarction compared with anterior circulation infarction. J Stroke Cerebrovasc Dis. 2015;24:1614-20.

6. Cui R, Qi Z, Zhou L, Li Z, Li Q, Zhang J. Evaluation of serum lipid profile, body mass index, and waistline in Chinese patients with type 2 diabetes mellitus. Clin Interv Aging. 2016;11:445-52.

7. Eckel RH, Grundy SM, Zimmet PZ. The metabolic syndrome. Lancet. 2005;365:1415-28.

8. Lee JS, Chang PY, Zhang Y, Kizer JR, Best LG, Howard BV. Triglyceride and $\mathrm{HDL}-\mathrm{C}$ dyslipidemia and risks of coronary heart disease and ischemic stroke by glycemic dysregulation status: the strong heart study. Diabetes Care. 2017;40:529-37.

9. Olamoyegun MA, Akinlade AT, Fawale MB, Ogbera AO. Dyslipidaemia as a riskfactor in the occurrence of stroke in Nigeria: prevalence and patterns. Pan Afr Med J. 2016;25:72.

10. Kanter JE, Johansson F, LeBoeuf RC, Bornfeldt KE. Do glucose and lipids exert independent effects on atherosclerotic lesion initiation or progression to advanced plaques? Circ Res. 2007;100:769-81.

11. Bogousslavsky J, Regli F. Centrum ovale infarcts: subcortical infarction in the superficial territory of the middle cerebral artery. Neurology. 1992;42:1992-8.

12. Shafrir E, Raz I. Diabetes: mellitus or lipidus? Diabetologia. 2003;46:433-40.

13. Luo Y, Li J, Zhang J, Xu Y. Low HDL cholesterol is correlated to the acute ischemic stroke with diabetes mellitus. Lipids Health Dis. 2014;13:171. 
14. Urbina EM, Khoury PR, McCoy CE, Dolan LM, Daniels SR, Kimball TR. Triglycerideto HDL-C ratio and increased arterial stiffness in children, adolescents, andyoung adults. Pediatrics. 2013;131:e1082-90.

15. Arboix A, Rivas A, García-Eroles L, de Marcos L, Massons J, Oliveres M. Cerebral infarction in diabetes: clinical pattern, stroke subtypes, and predictors of in-hospital mortality. BMC Neurol. 2005;5:9.

16. Karapanayiotides T, Piechowski-Jozwiak B, van Melle G, Bogousslavsky J, Devuyst G. Stroke patterns, etiology and prognosis in patients with diabetes mellitus. Neurology. 2004;62:1558-1562.11.

17. Pontiroli $A E$, Monti LD, Pizzini A, Piatti P. Familial clustering of arterial blood pressure, $\mathrm{HDL}$ cholesterol, and pro-insulin but not of insulin resistance and microalbuminuria in siblings of patients with type 2 diabetes. Diabetes Care 2000;23:1359-64.

18. Khavandi M, Duarte F, Ginsberg HN, Reyes-Soffer G. Treatment of dyslipidemias to prevent cardiovascular disease in patients with type 2 diabetes. Curr Cardiol Rep. 2017;19:7.

19. Semenkovich CF. Insulin resistance and atherosclerosis. J Clin Invest. 2006;116:1813-22.

20. Chapman MJ, Sposito AC. Hypertension and dyslipidaemia in obesity and insulin resistance: pathophysiology, impact on atherosclerotic disease and pharmacotherapy. Pharmacol Ther. 2008;117:354-73.

21. Ginsberg HN, Zhang YL, Hernandez-Ono A. A metabolic syndrome: focus on dyslipidemia. Obesity. 2006;14(Suppl):41S-9S.

22. Standards of Medical Care in Diabetes-2017. Summary of revisions. Diabetes Care. 2017;40(Suppl 1):S4-5.

23. Kim DE, Kim JY, Jeong SW, Cho YJ, Park JM, Lee JH, Kang DW, Yu KH, Bae HJ, Hong KS, Koo JS, Lee SH, Lee BC, Han MK, Rha JH, Lee YS, Kim GM, Chae SL, Kim JS, Kwon SU. Association between changes in lipid profiles and progression of symptomatic intracranial atherosclerotic stenosis: a prospective multicenter study. Stroke. 2012;43:1824-30.

24. Amarenco P, Goldstein LB, Messig M, O'Neill BJ, Callahan A 3rd, Sillesen $\mathrm{H}$, Hennerici MG, Zivin JA, Welch KM, SPARCL Investigators. Relative and cumulative effects of lipid and blood pressure control in the stroke prevention by aggressive reduction in cholesterol levels trial. Stroke. 2009:40:2486-92.

25. O'Donnell MJ, Xavier D, Liu L, Zhang H, Chin SL, Rao-Melacini P, Rangarajan S, Islam S, Pais P, McQueen MJ, Mondo C, Damasceno A, Lopez-Jaramillo P, Hankey GJ, Dans AL, Yusoff K, Truelsen T, Diener HC, Sacco RL, Ryglewicz D, Czlonkowska A, Weimar C, Wang X, Yusuf S, INTERSTROKE investigators. Risk factors for ischaemic and intracerebral haemorrhagic stroke in 22 Countries (the INTERSTROKE study): a case-control study. Lancet. 2010;376:112-23.

26. Andersson C, Lyass A, Vasan RS, Massaro JM, D'Agostino RB Sr, Robins SJ. Long-term risk of cardiovascular events across a spectrum of adverse major plasma lipid combinations in the Framingham heart study. Am Heart J. 2014;168:878-83.

27. Giannini C, Santoro N, Caprio S, Kim G, Lartaud D, Shaw M, Pierpont B, Weiss $\mathrm{R}$. The triglyceride-to-HDL cholesterol ratio: association with insulin resistance in obese youths of different ethnic backgrounds. Diabetes Care. 2011;34:1869-74.

28. Ng DS. Diabetic dyslipidemia: from evolving pathophysiological insight to emerging therapeutic targets. Can J Diabetes. 2013;37:319-26.

\section{Ready to submit your research? Choose BMC and benefit from:}

- fast, convenient online submission

- thorough peer review by experienced researchers in your field

- rapid publication on acceptance

- support for research data, including large and complex data types

- gold Open Access which fosters wider collaboration and increased citations

- maximum visibility for your research: over $100 \mathrm{M}$ website views per year

At BMC, research is always in progress.

Learn more biomedcentral.com/submissions 